\title{
Histological evidence of reparative activity in chorioamniotic membrane following open fetal surgery for myelomeningocele
}

\author{
NATALIA S. CARVALHO ${ }^{1,2}$, ANTONIO F. MORON ${ }^{1,2}$, RAMKUMAR MENON $^{3}$, SERGIO CAVALHEIRO $^{2,4}$, \\ MAURICIO M. BARBOSA ${ }^{1,2}$, HERBENE J. MILANI ${ }^{1,2}$ and MARCIA M. ISHIGAI ${ }^{5}$
}

\author{
${ }^{1}$ Department of Obstetrics, Escola Paulista de Medicina, Federal University of São Paulo, São Paulo, SP 04021-001; \\ ${ }^{2}$ Fetal Medicine Division, Hospital e Maternidade Santa Joana, São Paulo, SP 04103-000, Brazil; ${ }^{3}$ Department of Obstetrics \\ and Gynecology, University of Texas Medical Branch, Galveston, TX 77555, USA; Departments of ${ }^{4}$ Neurosurgery \\ and ${ }^{5}$ Pathology, Escola Paulista de Medicina, Federal University of São Paulo, São Paulo, SP 04021-001, Brazil
}

Received March 21, 2017; Accepted July 12, 2017

DOI: $10.3892 / \mathrm{etm} .2017 .4976$

\begin{abstract}
An increased understanding of the reparative process in fetal membrane following surgical techniques may be helpful to decrease the risks to mother and fetus and avoid adverse pregnancy outcomes. The present study discusses histological evaluation of the fetal membrane following open fetal surgery. Chorioamniotic membranes $(\mathrm{n}=10)$ were obtained following birth from pregnancies that underwent open fetal surgery for myelomeningocele. The collagen distribution was quantified using picrosirius-polarization method comparing the suture site with non-suture site. The differences between the collagen fiber percentages at the two sites was evaluated by the paired t-test with $\mathrm{P}<0.05$. The mean gestational age of fetal surgery was $26.09 \pm 0.3$ and $33.81 \pm 0.82$ weeks at birth. The picrosirius red sign was more intense at the suture site, primarily associated with collagen type 1 . Collagen observed in the surgical area was significantly increased $(13.22 \pm 2.84 \%)$ compared with the non-surgical area $(6.16 \pm 1.09 \%$; $\mathrm{P}<0.0001)$. It was observed that the reparative activity at the suture site of the fetal membrane was characterized by a significant increase in collagen fibers. The findings suggest nascent collagen synthesis, tissue remodeling and repair of suture site, a mechanism likely to prevent the amniotic fluid leakage and maintain pregnancy following open fetal surgery.
\end{abstract}

\section{Introduction}

Few studies have been conducted about the repair capacity of the fetal membrane with controversial results (1-6). It is believed that fetal membrane has no repair capacity, since it is

Correspondence to: Dr Natalia S. Carvalho, Department of Obstetrics, Escola Paulista de Medicina, Federal University of São Paulo, Av. Itapaiuna, 1800, Torre Amadryas, Apt 211, São Paulo, SP 04021-001 Brazil

E-mail: natcarvalho@msn.com

Key words: fetal membrane repair, fetal membrane healing, myelomeningocele, open fetal surgery, spina bifida not innervated or vascularized (7). Therefore, it is considered that they are unable to have an inflammatory response to tissue regeneration or repair.

Fetal membrane rupture after invasive procedures like amniocentesis; however, it is most often self-limited and repaired and resealed spontaneously $(8,9)$. Some cases of spontaneous membrane rupture, particularly at very early gestational ages (GAs), exhibit spontaneous resolution with amniotic fluid levels returning to normal (1), suggesting that the fetal membranes possess resealing capacity in their structure. This is corroborated by in vitro and animal model studies $(2,3,10,11)$.

In vitro studies shows that cells derived from the amnion are able to repair 75 to $80 \%$ of microsurgical defect in a period of $24 \mathrm{~h}$ (2). In a rabbit model of iatrogenic rupture of membranes, membrane integrity was restored a week after the rupture in $40 \%$ of cases (11). Levels of metalloproteinases (MMPs) 2 and 9 and tissue inhibitors of metalloproteinases (TIMPs) were increased in the amniotic fluid after one week of rupture, suggesting fetal membrane tissue remodeling process involving the activation of gelatinases (MMP 2 and 9) $(10,11)$.

On the other hand, no proliferation of fibroblasts and firbrous tissue was observed in human fetal membranes obtained from patients undergoing fetoscopy. The membranes were poorly viable, exhibiting picnosis, low cellularity and no spontaneous closure of the lesions was observed (6). In addition, histologic signs of healing was also not observed in trocar insertion sites of fetal membrane after fetal laser surgery. Apoptosis and collagen disorganization was observed in the amnion cells and extracellular matrix of he amnion (12).

Progress in fetal surgery, particularly using the open technique, has demonstrated increasing promise in the treatment of fetal anomalies. Despite of the risks and complications that are related of this procedure, the management of myelomeningocele study (MOMS) concluded that intrauterine surgical correction is currently the best form of treatment of this condition (13).

Due to restrictions of the government agency ANVISA, is not allowed to use staplers for performing open fetal surgery in Brazil. Since 2011, we have used an alternative surgical method for open fetal surgery developed by our 
team to correct myelomeningocele. This technique does not use staples and have similar results to those observed in the MOMS study (14). Therefore resealing of the membranes and avoiding membrane rupture associated complications are important prior to attempting fetal surgery. In most cases, after open fetal surgery, the amniotic fluid volume remains normal up to the time of fetal delivery $(77-79 \%)(13,14)$ and we have hypothesized that exist an active process of fetal membrane repair and not only a mechanical action of the surgical sutures in conjunction with the myometrial tissue reaction. Therefore, evaluation of the repair process in the fetal membrane after intrauterine surgical procedures is particularly important.

The aim of this study was to morphologically evaluate the suture site of fetal membrane after open fetal surgery for myelomeningocele and to compare the characteristics and composition of collagen between the suture site and non-suture site of the same membrane.

\section{Materials and methods}

This was an experimental study conducted on samples of fetal membranes, which were obtained after delivery of 10 fetuses that underwent intrauterine surgery for myelomeningocele (Fig. 1). We collected information associated with the patients and fetuses such as age, parity, GA at the time of fetal surgery and childbirth, time between fetal surgery and childbirth, fetal weight at birth, and complications after surgery. The study was approved by the institutional research ethics board (no. 887.954; November 18, 2014).

The placentae were rinsing briefly in water and examined according to the placental examination guideline (15). The surgical suture site and the rupture site of membranes were identified and analyzed separately, and the suture site was marked with ink (Fig. 2). Further, one or more membrane segments (average of two segments) were sectioned from the area of suture toward to the edge of placenta, so that most of the previously marked area was included. It was made a roll of membranes, which in turn was cross-sectioned and histologically processed (Fig. 2).

The routine study of the remaining sample (the membranes including the rupture area, umbilical cord, and placenta) followed the recommended guideline for normal placenta study (15).

Histological sections from all samples were made with a thickness of 4-5 $\mu \mathrm{m}$, obtaining one slice per slide and an average of 2 fragments. The sections were stained using hematoxylin-eosin for first morphological evaluation. In each case, one 5- $\mu \mathrm{m}$ thick histological slice, which was most representative of the area of suture, was selected for picrosirius red staining.

The morphological pattern observed in the membrane suture site was compared with that found in the non-suture site of membrane. We evaluated the following characteristics: the architecture of the amnion, chorion, and decidua; the intensity and type of collagen; and the presence of necrosis/degeneration.

The collagen was quantified using picrosirius red staining, visualized under circular polarized microscopy. The picrosirius red is a strong linear anionic dye which contains six sulfonic radicals that react with basic groups present in the collagen molecules promoting an enhancement of its normal birefringence under cross-polarized light. This effect is due to the parallel arrangement of its molecules with the long axes of collagen fibers. This method is specific for collagenous structures and allows detection of small amounts of collagen and the differentiation of their types. The collagen type I shows up as thick, strongly birefringent, yellow or red fibers while the collagen type III displays the form of thin, weakly birefringence, greenish fibers. The collagen type II presents a weak birefringence of varying color.

The sections from each paraffin block were submitted to the picrosirus staining as previously described (16). Briefly, all the sections were deparaffinized, hydrated and then stained during $1 \mathrm{~h}$, with a $0,1 \%$ solution of sirius red (F3BA) in saturated aqueous picric acid. This was followed by rising with distilled water, dehydration and mounting.

A conventional optical microscope (Olympus BX40 equipped with two polaroid filters) was used for examination of the sections. These two filters were aligned to that the background in the field of view was as dark as possible. The digitalized images were recorded using an camera (Q-Color3; Olympus America Inc., Melville, NY, USA), resolution 200 dpi.

Ten fields/slides were examined (5 fields each of the suture and normal areas) at a x400 magnification, and the captured images were stored as TIFF files for subsequent quantification of the percentage of stained area as compared to the total area of the digitized field using UTHSCSA Image Tool 3.0 software (University of Texas Health Science Center, San Antonio, TX, USA). Villous remnants and deciduous vessels were avoided during image acquisition. Fibrous villi and amniotic epithelium were considered as positive and negative controls respectively. The results were compared using statistical analysis.

Descriptive analysis of the variables followed a roughly normal distribution. The paired t-test was used to evaluate the difference in percentage of collagen between the suture site and normal membrane site distant from the sutures. All data was stored in Excel for MAC 2011 spreadsheets (Microsoft, Redmond, WA, USA) and analyzed using SPSS software version 15.0 for Windows (SPSS, Inc., Chicago, IL, USA).

\section{Results}

The mean age of the pregnant women was 29.00 \pm 5.52 years, and $80 \%$ were nulliparous. The mean GA at the time of fetal surgery was $26.09 \pm 0.3$ weeks and GA at birth was $33.81 \pm 0.82$ weeks $(32-36$ weeks). The time between fetal surgery and childbirth was 54.01 \pm 13.39 days (Table I).

The cesarean section was performed due to ultrasonographic modifications of uterine scar (5), elective delivery after 36 weeks (2), premature rupture of membranes (1), oligohydramnios (1), and preterm labor (1). The newborns weight was 2,286 $\pm 381 \mathrm{~g}(1,690-2,800 \mathrm{~g})$ and all had normal Apgar score. The placentas weighed $366 \pm 79$ grams, with a thickness of $2.43 \pm 0.68 \mathrm{~cm}$ and a diameter of $15.45 \pm 1.53 \mathrm{~cm}$. All placentas showed appropriate villous maturation for third trimester gestation, and all umbilical cords exhibited three vessels, with mean thickness of $1.4 \pm 0.2 \mathrm{~cm}$ and eccentric insertion (Table I).

Macroscopic evaluation showed increased thickness of the membrane in the suture area compared with normal 
Table I. Clinical and pathological findings of the 10 placentas after open fetal surgery for myelomeningocele.

\begin{tabular}{lc}
\hline Variables & Average \pm SD \\
\hline Clinical findings & \\
Maternal age & $29.00 \pm 5.52$ \\
Parity & $1.00 \pm 0.44$ \\
Gestational age during fetal surgery & $26.09 \pm 0.30$ \\
Gestational age in birth & $33.81 \pm 0.82$ \\
Time between fetal surgery and childbirth & $54.01 \pm 13.39$ \\
Newborn weight & $2286 \pm 381$ \\
Macroscopy & \\
Placental weight & $366 \pm 79.05$ \\
Placental thickness & $2.43 \pm 0.68$ \\
Placental diameter & $15.45 \pm 1.53$ \\
Thickness of umbilical cord & $1.40 \pm 0.20$ \\
Histology & \\
Collagen evaluation (picrosirius red) & \\
Suture area & $13.22 \%$ \\
Non-suture area & $6.16 \%$ \\
\hline
\end{tabular}

${ }^{a} \mathrm{P}<0.0001 . \mathrm{SD}$, standard deviation.

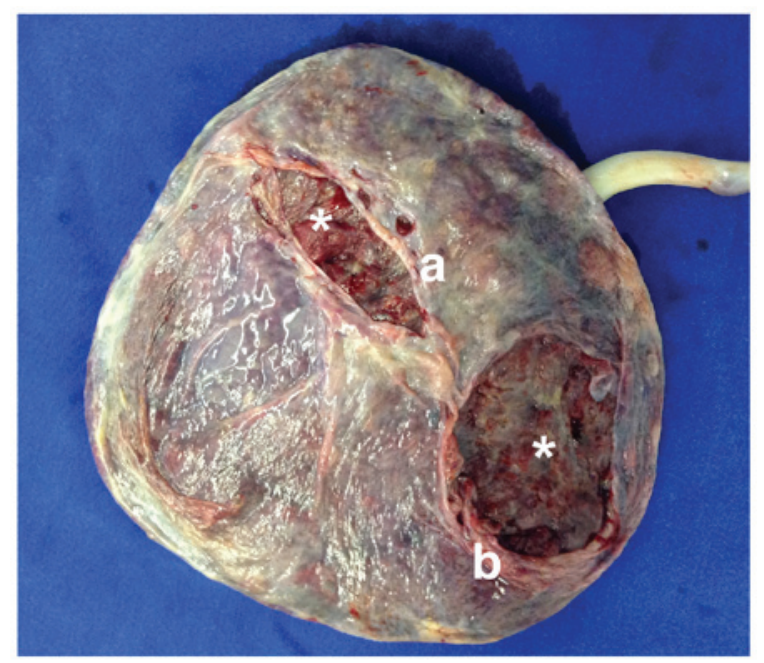

Figure 1. Macroscopic aspects of the placenta after surgical removal. The membranes are covering the basal plate $\left(^{*}\right)$ and present two holes bordered by (b) rupture area and (a) suture area.

areas of the same membrane. The suture areas revealed holes of different sizes with regular smooth and thickened edge. Histological analysis of sections stained with hematoxylin-eosin showed fibrous thickening and loss of normal membrane architecture in the area of surgery with fusion and poor delimitation between amnion and chorion in all samples (Figs. 3 and 4).

At the edge of the suture, the accumulation of collagen tissue in some places appeared as a nodular structure in which the amniotic epithelium was degenerated or missing. Moving away from the suture area, the epithelium and membrane as a whole assumed its usual morphology. Some foci of
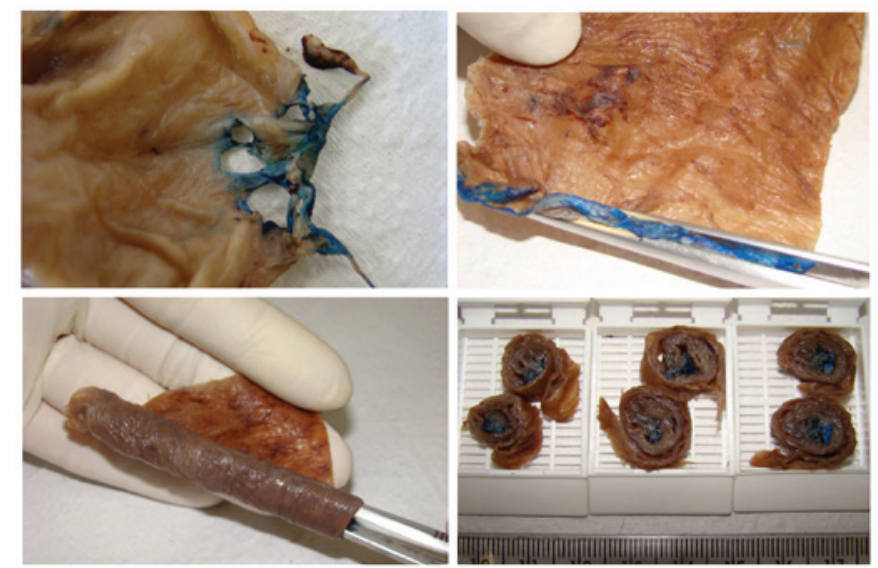

Figure 2. Prepare process of fetal membranes. The surgical suture site was identified and marked with ink. It was made a roll of membranes, which was cross-sectioned and histologically processed.

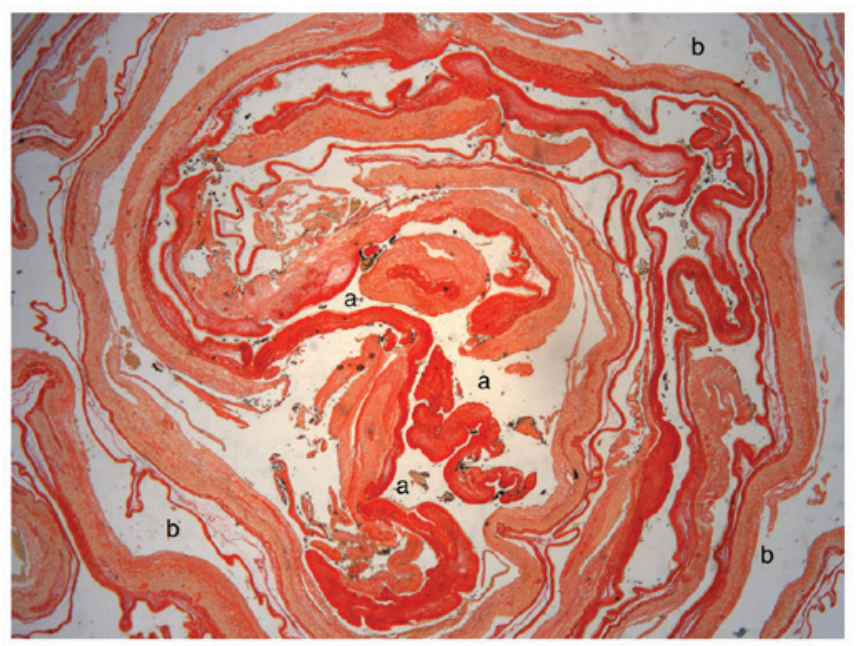

Figure 3. Fetal membrane histologic aspect (hematoxylin and eosin; magnification, $\mathrm{x} 2$ ). At the center of the image (a), it is observed the suture site with proliferation of fibrous tissue and degeneration of the amniotic epithelium. Moving away from the suture area (b), the epithelium and membrane assumed its usual morphology.

choriodecidual necrosis were observed both in the surgical suture region and ruptured area of the fetal membrane.

Sections stained with picrosirius red showed more expressive birefringence, viewed by polarized light, in the suture areas and demonstrated predominance of type 1 collagen (Fig. 5). The mean percent collagen in the surgical area was $13.22 \pm 2.84 \%$ and in the normal membrane area this value was $6.16 \pm 1.09 \%(\mathrm{P}<0.0001$; Table I). The mean difference between the percentage of collagen in the suture area and the normal area of the membrane was 7\% [95\% confidence interval (4.9-9.2)] with greater variability in the area of the suture.

\section{Discussion}

We examined resealing capability of human fetal membranes through collagen mediated repair process after fetal surgery in cases with myelomeningocele. We report a repair process in the fetal membranes represented by a significant increase 

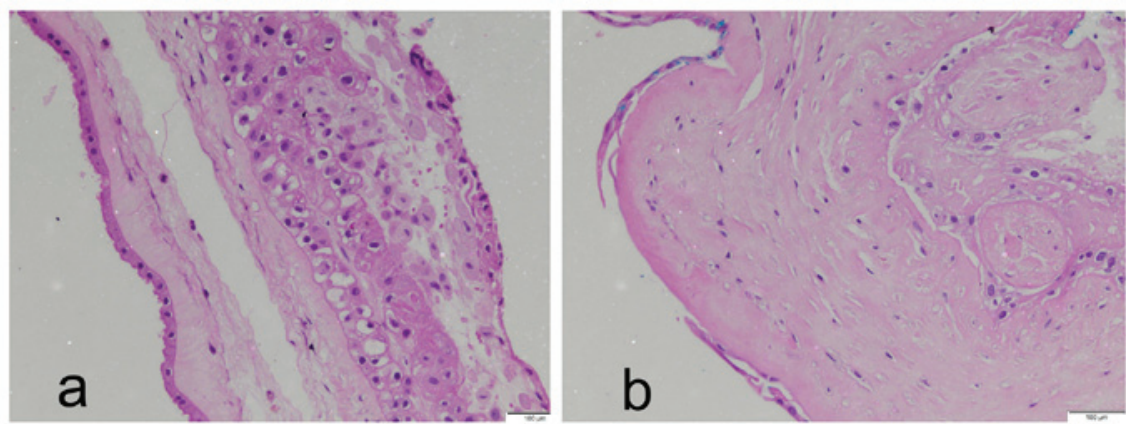

Figure 4. Comparison of fetal membrane histologic aspect between two groups (hematoxylin and eosin; magnification, $\mathrm{x} 400$ ). In the normal area of membrane (a) the morphological structure is maintained. In the suture area, (b) the membrane is thickened by fibrous tissue. Amnion and chorion are fused and disorganized showing areas of degeneration and loss of amniotic epithelium.
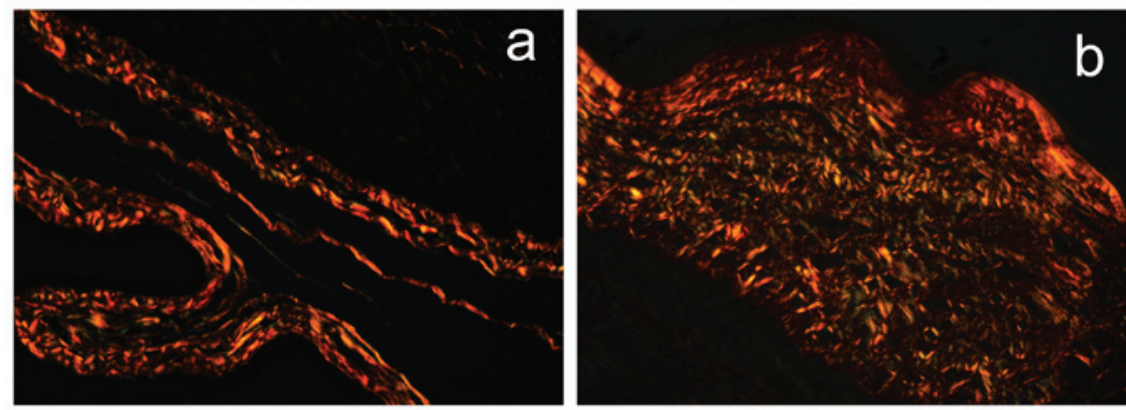

Figure 5. Comparison of picrosirius red between two groups, observed under polarized light (magnification, x400). An increase of collagen fibers can be observed in the suture site (b) when compared with the normal membrane site (a). It shows a predominance of type I collagen appearing as bright yellow-red/orange fibers.

in collagen fibers in the membrane at the suture site compared with normal site of the same membrane.

The repair process in the fetal membrane may be one of the mechanisms that limit the amniotic fluid leakage and permit the maintenance of gestation after open fetal surgery.

Similar results were observed in an experimental model that evaluated the response of the fetal membrane after needle perforation of the amnion in female rats at 15 or 16 days of gestation (17). There was thickening of the wound borders with intense fibroblast proliferation that progressed with significant reduction of the wound opening between the membranes on day 5 after the procedure (17).

In vitro studies showed that cells obtained from the amnion lining are able to repair $75-80 \%$ of the central microsurgical wound within $24 \mathrm{~h}$ (2). In vitro repair capacity is dependent on GA. Cells obtained at an earlier GA had a higher proliferation rate, and were able to close the central wound more rapidly (3).

However, in humans, there are no reports of the closure of surgical wounds after iatrogenic injury. Study by Gratacós et al did not observe spontaneous closure of the chorioamniotic membrane after fetoscopy in 15 pregnant women with mean GA of 22.2 weeks (6). In this study, unlike our cases, fibrosis was also not observed. Morphological analysis, which was conducted on an average of 60 days after surgery, showed large holes with smooth edges, which were unconnected to each other in the amnion and chorion. The anti-human Ki-67, the anti-human cytokeratin antibody and the anti-human vimentin antibody was analyzed and showed that fibroblast proliferation was discrete and limited to the chorion.
The observed differences can be partially addressed based on our distinct approaches. In our study we used the open fetal surgery technic that consist of an opening of approximately $6 \mathrm{~cm}$ in utero and fetal membrane. After surgery this opening is closed in three layers and the fetal membrane is attached to the myometrium. We analyzed fibrosis in the defect area through the picrosirius red staining that allows to quantify the amount of collagen. We also compared the collagen in the defect site with no-defect site of the same membrane.

It is possible that the different finding is attributed to the nature of the procedure during the gestation. In Gratacos study, the membrane defect was produced by fetoscopy and despite its small size, the lack of proximity between the edges of defect throughout the pregnancy favoring the dissociation of the choriodecidual and amniotic layers. Although this may have contributing to decrease the functional defect size, this effect may have acted as limiting factor in the healing process involving the decidua and chorioamniotic membrane.

In contrast, in our study, the fetal membrane was initially sutured to the internal third of myometrium using continuous suture of 4-0 monocryl around the whole incision. The second suture was performed with 2-0 Vicryl including the myometrium and amniotic membrane closing completely the opening, preventing amniotic fluid leakage and promoting to the fibrous repair.

The following mechanisms may be involved in closing the surgical area: the shift between the amnion and chorion which dissociates the holes in each of these membranes $(6,18)$, the 
retraction of the surgical wound through myometrial contraction, scarring in the myometrial and decidual layers, or a repair process in the fetal membrane itself (17).

Although we did not observe complete healing of the membranes and the histological examination did not show evident mitotic activity as demonstrated by the lack mitotic figures on H\&E staining, and the rare positivity to Ki67 marker previously observed in one of our cases, the significant amount of collagen at the surgical area indicates that it occurred in early stages of the postoperative period as already observed (17).

There are several mechanisms involved in the production of fibrosis besides the fibroblast proliferation. Reaction to tissue damage increases an inflammatory process that involved immune cell migration (neutrophils and macrophages) and local production of MMPs. This tissue remodeling process increases amount of collagen type 1 and 3 to plug the rupture site.

During the fibrogenesis, myofibroblastic cells secret extracellular matrix protein. These cells are found to be originated from preexistent fibroblast but also from the mechanism of epithelial-mesenchymal transition in which amniotic cells may assume a mesenchymal phenotype under the influence of injurious event (19). The fibrosis and the epithelial-mesenchymal transition are mainly regulated by transforming grown factor $\beta$ (TGF $\beta$ ), a multifunctional cytokine that has a central role in tissue repair. TGF $\beta$ is produced by macrophages and other cells that are present in chorioamniotic membranes and decidua giving to them the potential to tissue repair. We do not rule out epithelial-mesenchymal transition in this rea that promotes cell cycle, invasion and ECM building. Although, our staining are not designed to address such a transition.

The development of tissue fibrosis depends on the balance between the deposition and degradation of the extracellular matrix, which is modulated by the action of MMPs and their inhibitors (TIMPs) (20). Prior reports have shown that MMP 2 and 9 and TIMPs that are responsible for type 3 collagen turnover were increased in the basement membrane after intrauterine procedures in rabbits, suggesting an active mechanism for fetal membrane repair through collagen turnover at the wound site (11).

In summary, we demonstrate a collagen type I mediated repair process of the chorioamniotic membrane on the operative wound. An inflammatory tissue remodeling response associated with surgical repair may contribute to maintenance of pregnancy and amniotic fluid volume after open fetal surgery.

Further studies are required to better understand the healing process in the chorioamniotic membrane after fetal surgery in order to help reduce prematurity, premature rupture of membranes and oligohydramnios, which are the most important complications of intrauterine procedures. Understanding the reparative process in the fetal membrane may be helpful to improve the fetal surgery results in an effort to decrease the risks to mother and fetus.

\section{Acknowledgements}

We would like to thank the Coordenação de Aperfeiçoamento de Pessoal de Nível Superior-CAPES, Brazil, for a PhD scholarship received by NSC.

\section{References}

1. Johnson JW, Egerman RS and Moorhead J: Cases with ruptured membranes that 'reseal'. Am J Obstet Gynecol 163: 1024-1032, 1990.

2. Quintero RA, Carreño CA, Yelian F and Evans MI: Repair kinetics of amnion cells after microsurgical injury. Fetal Diagn Ther 11: 348-356, 1996.

3. Bilic G, Ochsenbein-Kölble N,Hall H,Huch R and Zimmermann R: In vitro lesion repair by human amnion epithelial and mesenchymal cells. Am J Obstet Gynecol 190: 87-92, 2004.

4. Devlieger R, Millar LK, Bryant-Greenwood G, Lewi L and Deprest JA: Fetal membrane healing after spontaneous and iatrogenic membrane rupture: A review of current evidence. Am J Obstet Gynecol 195: 1512-1520, 2006.

5. Devlieger R, Gratacós E, Verbist L, Pijnenborg R and Deprest J: Gestational age-dependent repair kinetics of microsurgical defects in monolayers of human amniocytes. Gynecol Obstet Invest 69: 62-66, 2010.

6. Gratacós E, Sanin-Blair J, Lewi L, Toran N, Verbist G, Cabero L and Deprest J: A histological study of fetoscopic membrane defects to document membrane healing. Placenta 27: 452-456, 2006.

7. Bryant-Greenwood GD: The extracellular matrix of the human fetal membranes: Structure and function. Placenta 19: 1-11, 1998.

8. Rocker I and Laurence KM: Defect in fetal membranes after fetoscopy. Lancet 1: 716, 1978.

9. Borgida AF, Mills AA, Feldman DM, Rodis JF and Egan JF: Outcome of pregnancies complicated by ruptured membranes after genetic amniocentesis. Am J Obstet Gynecol 183: 937-939, 2000.

10. Devlieger R, Riley SC, Verbist L, Leask R, Pijnenborg R and Deprest JA: Matrix metalloproteinases-2 and -9 and their endogenous tissue inhibitors in tissue remodeling after sealing of the fetal membranes in a sheep model of fetoscopic surgery. J Soc Gynecol Investig 9: 137-145, 2002.

11. Devlieger R, Deprest JA, Gratacós E, Pijnenborg R, Leask R and Riley SC: Matrix metalloproteinases -2 and -9 and their endogenous tissue inhibitors in fetal membrane repair following fetoscopy in a rabbit model. Mol Hum Reprod 6: 479-485, 2000.

12. Papanna R, Mann LK Moise KJ Jr, Kyriakides T, Johnson A, Garcia E, Buhimschi CS and Buhimschi IA: Histologic changes of the fetal membranes after fetoscopic laser surgery for twin-twin transfusion syndrome. Pediatr Res 78: 247-255, 2015.

13. Adzick NS, Thom EA, Spong CY, Brock JW III, Burrows PK, Johnson MP, Howell LJ, Farrell JA, Dabrowiak ME, Sutton LN, et al: A randomized trial of prenatal versus postnatal repair of myelomeningocele. N Engl J Med 364: 993-1004, 2011.

14. Moron A, Barbosa M, Milani H, Hisaba W, Carvalho N and Cavalheiro S: 771: Short-term surgical and clinical outcomes with a novel method for open fetal surgery of myelomeningocele. Am J Obstet Gynecol 212: S374, 2015.

15. Langston C, Kaplan C, Macpherson T, Manci E, Peevy K, Clark B, Murtagh C, Cox S and Glenn G: Practice guideline for examination of the placenta: Developed by the pathology practice guideline development task force of the college of American pathologists. Arch Pathol Lab Med 121: 449-476, 1997.

16. Calvi EN, Nahas FX, Barbosa MV, Calil JA, Ihara SS, Silva Mde S, Franco MF and Ferreira LM: An experimental model for the study of collagen fibers in skeletal muscle. Acta Cir Bras 27: 681-686, 2012.

17. Sopher D: The response of rat fetal membranes to injury. Ann R Coll Surg Engl 51: 240-249, 1972.

18. Behzad F, Dickinson MR, Charlton A and Aplin JD: Brief communication: Sliding displacement of amnion and chorion following controlled laser wounding suggests a mechanism for short-term sealing of ruptured membranes. Placenta 15: 775-778, 1994.

19. Romero-Valdovinos M, Bobadilla-Sandoval N, Flisser A and Vadillo-Ortega F: The epithelial mesenchymal transition process may contribute to the pathogenesis of amniotic band syndrome. Med Hypotheses 83: 306-311, 2014.

20. Qin X, Garibay-Tupas J, Chua PK, Cachola L and Bryant-Greenwood GD: An autocrine/paracrine role of human decidual relaxin. I. Interstitial collagenase (matrix metalloproteinase-1) and tissue plasminogen activator. Biol Reprod 56: 800-811, 1997. 